\title{
Parton distributions in a constituent quark scenario
}

\author{
S. Scopetta ${ }^{\mathrm{a}}$, V. Vento ${ }^{\mathrm{a}}$ and M. Traini ${ }^{\mathrm{b}}$ \\ a Departament de Fisica Teòrica, Universitat de València, 46100 Burjassot (València), \\ Spain; and Institut de Física Corpuscular, CSIC \\ b Dipartimento di Fisica, Università di Trento, I-38050 Povo (Trento), Italy, and INFN, \\ Gruppo Collegato di Trento
}

A simple picture of the constituent quark as a composite system of point-like partons is used to construct the unpolarized and polarized parton distributions by a convolution between constituent quark momentum distributions and constituent quark structure functions. We achieve good agreement with experiments in the unpolarized, as well as, in the polarized case. When our results are compared with similar calculations using non-composite constituent quarks, the accord with the experiments of the present scheme is impressive. We conclude that DIS data are consistent with a low energy scenario dominated by composite constituents of the nucleon.

At low energies, the so called naive quark model accounts for a large number of experimental observations. At large energies, $Q C D$ sets the framework for an understanding of the Deep Inelastic Scattering (DIS) phenomena beyond the Parton Model. However, the perturbative approach to $Q C D$ does not provide absolute values for the observables. The description based on the Operator Product Expansion (OPE) and the $Q C D$ evolution requires the input of non-perturbative matrix elements. We have developed an approach which uses model calculations for the latter ingredients at a low, hadronic, scale [1]. Moreover, in order to relate the constituent quark with the current partons of the theory, a procedure, hereafter called ACMP, has been applied [2, 3]. Within this approach, constituent quarks are effective particles made up of point-like partons (current quarks, antiquarks and gluons), interacting by a residual interaction described as in a quark model. The hadron structure functions are obtained by a convolution of the constituent quark model wave function with the constituent quark structure function. This idea has been recently used to successfully estimate the pion structure function [4. We summarize here our application to the unpolarized [3] and polarized [5] DIS off the nucleon.

In our picture the constituent quarks are themselves complex objects whose structure functions are described by a set of functions $\Phi_{a b}$ that specify the number of point-like partons of type $b$, which are present in the constituents of type $a$ with fraction $x$ of its total momentum [2, 3]. In general $a$ and $b$ specify all the relevant quantum numbers of the

\footnotetext{
*Supported in part by DGICYT-PB97-1227 and TMR programme of the European Commission ERB FMRX-CT96-008
} 
partons, i.e., flavor and spin. Let us discuss first the unpolarized case for the proton [3]. The functions describing the nucleon parton distributions omitting spin degrees of freedom are expressed in terms of the independent $\Phi_{a b}(x)$ and of the constituent probability distributions $u_{0}$ and $d_{0}$, at the hadronic scale $\mu_{0}^{2}$ [1], as

$f\left(x, \mu_{0}^{2}\right)=\int_{x}^{1} \frac{d z}{z}\left[u_{0}\left(z, \mu_{0}^{2}\right) \Phi_{u f}\left(\frac{x}{z}, \mu_{0}^{2}\right)+d_{0}\left(z, \mu_{0}^{2}\right) \Phi_{d f}\left(\frac{x}{z}, \mu_{0}^{2}\right)\right]$

where $f$ labels the various partons, i.e., valence quarks $\left(u_{v}, d_{v}\right)$, sea quarks $\left(u_{s}, d_{s}, s\right)$, sea antiquarks $(\bar{u}, \bar{d}, \bar{s})$ and gluons $g$. The different types and functional forms of the structure functions for the constituent quarks are derived from three very natural assumptions [2]:

i) The point-like partons are the quarks, antiquarks and gluons described by $Q C D$; ii) Regge behavior for $x \rightarrow 0$ and duality ideas; iii) invariance under charge conjugation and isospin. These considerations define the following structure functions [2]

$\Phi_{q f}\left(x, \mu_{0}^{2}\right)=C_{f} x^{a_{f}}(1-x)^{A-1}$,

where $f=q_{v}, q_{s}, g$ for the valence quarks, the sea and the gluons, respectively. Regge phenomenology suggests: $a_{q_{v}}=-0.5$ ( $\rho$ meson exchange) and $a_{q_{s}}=a_{g}=-1$ (pomeron exchange). The other ingredients of the formalism, i.e., the probability distributions for each constituent quark, are defined according to the procedure of ref. [1] and shown in [3]. Our last assumption relates to the hadronic scale $\mu_{0}^{2}$, i.e., that at which the constituent quark structure is defined. We choose $\mu_{0}^{2}=0.34 \mathrm{GeV}^{2}$, as defined in Ref. [1], namely by fixing the momentum carried by the various partons. This choice of the hadronic scale determines all the parameters except one, which is fixed through the data [3]. To complete the process, the above input distributions are NLO-evolved in the DIS scheme to the experimental scale, where they are compared with the data.

We next generalize our previous discussion to the polarized parton distributions. As it is explained in ref. [5], using $S U(6)$ (spin-isospin) symmetry and other reasonable simplifying assumptions, it can be shown that

$\Delta f\left(x, \mu_{0}^{2}\right)=\int_{x}^{1} \frac{d z}{z}\left[u_{0}\left(z, \mu_{0}^{2}\right) \Delta \Phi_{u f}\left(\frac{x}{z}, \mu_{0}^{2}\right)+d_{0}\left(z, \mu_{0}^{2}\right) \Delta \Phi_{d f}\left(\frac{x}{z}, \mu_{0}^{2}\right)\right]$,

where $f$ labels the various partons; it means that the $A C M P$ procedure can be extended to the polarized case just by introducing three additional structure functions for the constituent quarks: $\Delta \Phi_{q q_{v}}, \Delta \Phi_{q q_{s}}$ and $\Delta \Phi_{q g}$. In order to determine them we add two minimal assumptions: $i v$ ) factorization: $\Delta \Phi$ cannot depend upon the quark model used; v) positivity: the constraint $\Delta \Phi \leq \Phi$ is saturated for $x=1$. In such a way we determine completely the $\triangle \Phi$ 's. In fact, the $Q C D$ partonic picture, Regge behavior and duality imply that

$\Delta \Phi_{q f}=\Delta C_{f} x^{-\Delta a_{f}}(1-x)^{\Delta A_{f}-1}$

and $-\frac{1}{2}<\Delta a_{f}<0$, for all $f=q_{v}, q_{s}, g$, as allowed by dominant exchange of the $A_{1}$ meson trajectory [7]. Moreover, the assumption that the positivity restriction is saturated for $x=1$, in the spirit of ref. [6], implies that the $\Phi^{\prime} s$ and the $\Delta \Phi^{\prime} s$ have the same large $x$ behavior, and that $\Delta C_{f}=C_{f}$, (the latter being introduced in (2)); it means that the 

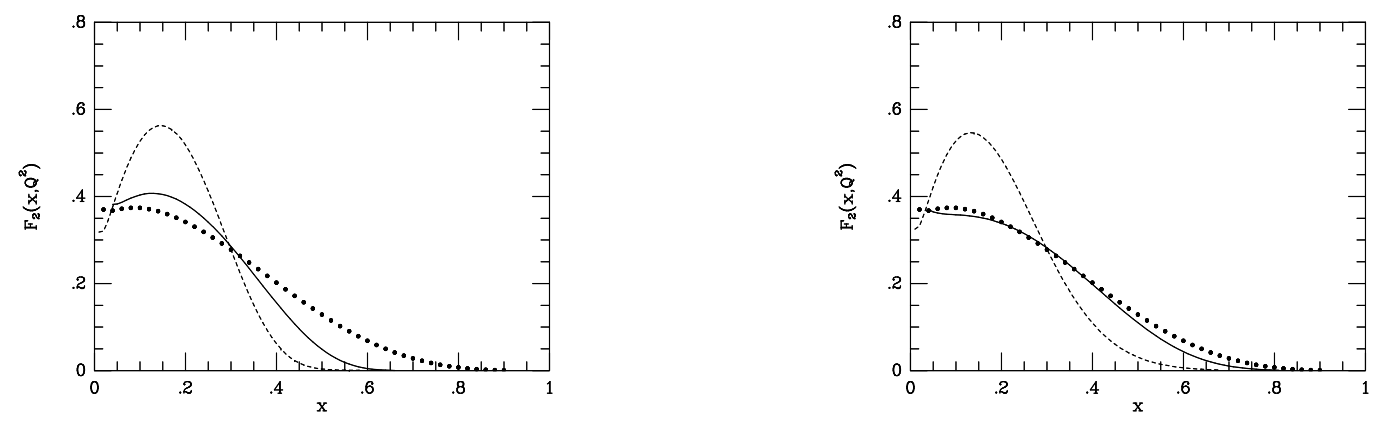

Figure 1. The proton $F_{2}\left(x, Q^{2}\right)$, obtained by NLO-evolution to $Q^{2}=10 \mathrm{GeV}^{2}$ (full), compared to the data (dots) [11]. The result which would be obtained disregarding the constituent structure is also shown (dashed). Left (right) panel: constituent wave functions form ref. [9] (ref. [10]).
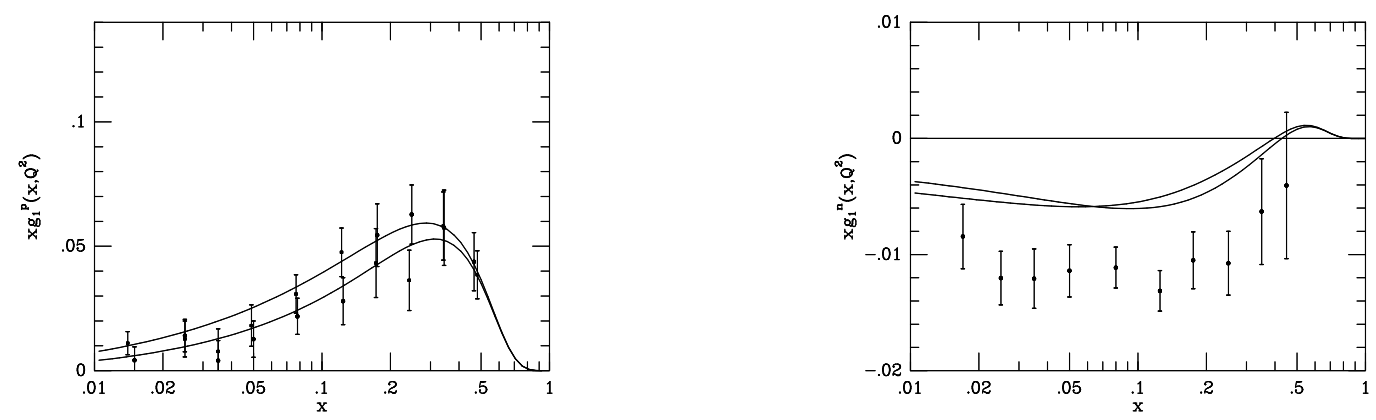

Figure 2. Left (Right): $x g_{1}\left(x, Q^{2}\right)$ for the proton (neutron) evolved at NLO to $Q^{2}=10$ (5) $\mathrm{GeV}^{2}$, for the two extreme Regge behaviors mentioned in the text (full curves). The wave functions used are from ref. [10]. The data [11] are shown for comparison.

partons which carry all of the momentum also carry all of the polarization. Let us stress that the change between the polarized functions and the unpolarized ones comes only from Regge behavior; as a matter of fact, it turns out that, except for the exponent $\Delta a_{f}$ shown above, the $\Delta \Phi$ 's, Eq. (田), are given by the unpolarized functions, Eq. (2). The other ingredients, i.e., the polarized distributions for each constituent quark, are defined according to the procedure of ref. [1] and they are shown in ref. [0]. Finally, the parton distributions at the hadronic scale are evolved to the experimental scale by performing a NLO evolution in the AB scheme [8]. Results are shown in Figs. 1 and 2. The structure function $F_{2}\left(x, Q^{2}\right)$, calculated by means of the obtained distributions in the DIS evolution scheme, is shown in Fig. 1. We see that data are successfully described, the agreement becoming impressive when compared with that obtained by similar calculations with noncomposite constituents.

Taking into account the almost inexistent fit of parameters, the results are surprisingly good for both models 09.10]. In particular the momentum distribution of ref. [10] seems to have been defined to fit the DIS data, which is not the case. In the polarized case, it is 
found [5] that the constituent structure functions Eq. (4) give a good result for the proton, but they fail in reproducing the recent precise neutron data. This is to be ascribed to our naive input for the sea and to the symmetry for the $u$ and $d$ quarks [5]. In particular, it has been shown that, by redefining the sea $\Delta \Phi$, changing only one parameter so that the experimental sea polarization is recovered, also the neutron is rather well described. Fig. 2 refers to this last scenario. We have traced back the remaining disagreement with the neutron data to the symmetric treatment of $u$ and $d$ quarks. It can be shown that a weak breaking of the SU(6) symmetry in the quark model used [10] and/or in the constituent quark structure functions improves considerably the agreement. The neutron is extremely sensitive to small changes in the valence structure.

The procedure is also able to predict successfully several observables, such as the nucleon axial charges [5]. It should be noticed that in this framework the spin crisis, as initially presented, does not arise.

Since the method seems to be predictive, we are confident that it could be useful to phenomenologically estimate unmeasured quantities, such as the transversity parton distribution $h_{1}$ [13] or off-forward quantities [14], which should be measured by the next generation of DIS experiments [15].

Summarizing, low energy models seem to be consistent with DIS data when a structure for the constituent is introduced. The crucial role played by the sea in the polarized case, as well as the implementation of Chiral Symmetry Breaking in our procedure, have to be more deeply investigated. It will be the subject of future work.

\section{Acknowledgements}

S.S. thanks the Organizers for the invitation and finantial support.

\section{REFERENCES}

1. M. Traini, V. Vento, A. Mair and A. Zambarda, Nucl. Phys. A 614 (1997) 472.

2. G. Altarelli, N. Cabibbo, L. Maiani and R. Petronzio Nucl. Phys. B 69 (1974) 531.

3. S. Scopetta, V. Vento and M. Traini, Phys. Lett. B 421 (1998) 64.

4. G. Altarelli, S. Petrarca and F. Rapuano, Phys. Lett. B 373 (1996) 200.

5. S. Scopetta, V. Vento and M. Traini, Phys. Lett. B 442 (1998) 28.

6. R. Carlitz and J. Kaur, Phys. Rev. Lett. 38 (1977) 673.

7. G. Altarelli, R.D. Ball, S. Forte and G. Ridolfi, Nucl. Phys. B 496 (1997) 337.

8. R.D. Ball, S. Forte and G. Ridolfi, Phys. Lett. B 378 (1996) 255; G. Ridolfi, this Workshop.

9. N. Isgur and G. Karl, Phys. Rev. D 18 (1978) 4187.

10. R. Bijker, F. Iachello and A. Leviatan, Ann. Phys. 236 (1994) 69.

11. EMC, Nucl. Phys. B328 (1989) 1; SMC, Phys. Rev. D 56 (1997) 5330; E154, Phys. Rev. Lett. 79 (1997) 26.

12. H. L. Lai et al., Phys. Rev. D 51 (1995) 4763.

13. V. Barone, this Workshop.

14. A. Radyushkin, this Workshop.

15. E. de Sanctis, this Workshop; T. Walcher, this Workshop. 